\title{
Inline myocardial t2* mapping with iterative robust fitting
}

\author{
Saurabh Shah ${ }^{1 *}$, Hui Xue ${ }^{2}$, Andreas Greiser ${ }^{3}$, Peter Weale ${ }^{1}$, Taigang He ${ }^{4}$, David N Firmin ${ }^{4}$, Dudley J Pennell ${ }^{4}$, \\ Sven Zuehlsdorff', Jens Guehring ${ }^{2}$
}

From 2011 SCMR/Euro CMR Joint Scientific Sessions

Nice, France. 3-6 February 2011

\section{Introduction}

Myocardial T2* measurement is a valuable tool for non-invasive assessment of iron overload, and is clinically employed for planning and monitoring ironchelating treatments for transfused thalassemia major patients [1]. Presently, for T2* assessment, dark-blood prepared gradient echo (GRE) images are acquired at multiple echo times (TEs). Thereafter, these images are analyzed within offline software such as CMRTools: ThalassaemiaTools ${ }^{\circ}$, in which the septal signal of a full thickness ROI is fitted to a monoexponential decay curve to estimate myocardial T2* [2]. The goal of this study was to develop and test a $\mathrm{T} 2 \%$ measurement technique with automated inline T2*-map generation. Availability of such a technique on commercial MR systems may further utilization of such measurements in this patient group.

\section{Methods}

An ECG-triggered 2D multi-echo GRE sequence was implemented on a $1.5 \mathrm{~T}$ MR scanner (MAGNETOM Espree, Siemens AG) with support for dark-blood preparation. To generate an inline $\mathrm{T}^{*}$-map, an integrated image reconstruction performs pixel-wise $\mathrm{T} 2^{*}$ estimation using a robust fit, in which the signal at each TE is iteratively weighted to reflect its fidelity to monoexponential decay curve. Points farther from the ideal relaxation curve are weighted lower, reducing their influence on the fit.

In five healthy volunteers, the method was used to acquire short axis images of the heart, accompanied by inline $\mathrm{T} 2 \%$-map computation. Additionally, to compare the accuracy of the robust-fit with a validated method, $\mathrm{T} 2 *$ maps were retrospectively computed using multi-echo

${ }^{1}$ Siemens Healthcare, Chicago, IL, USA

Full list of author information is available at the end of the article images of 32 patients. In all cases, a septal region-ofinterest was manually drawn to obtain an average T2* value.

\section{Results}

Fig. 1 shows the T2*-maps obtained in two volunteers. Fig. 2 demonstrates $\mathrm{T} 2 *$ estimates using the inline $\mathrm{T} 2{ }^{*}$ maps in 3 patients with suspected iron overload, which

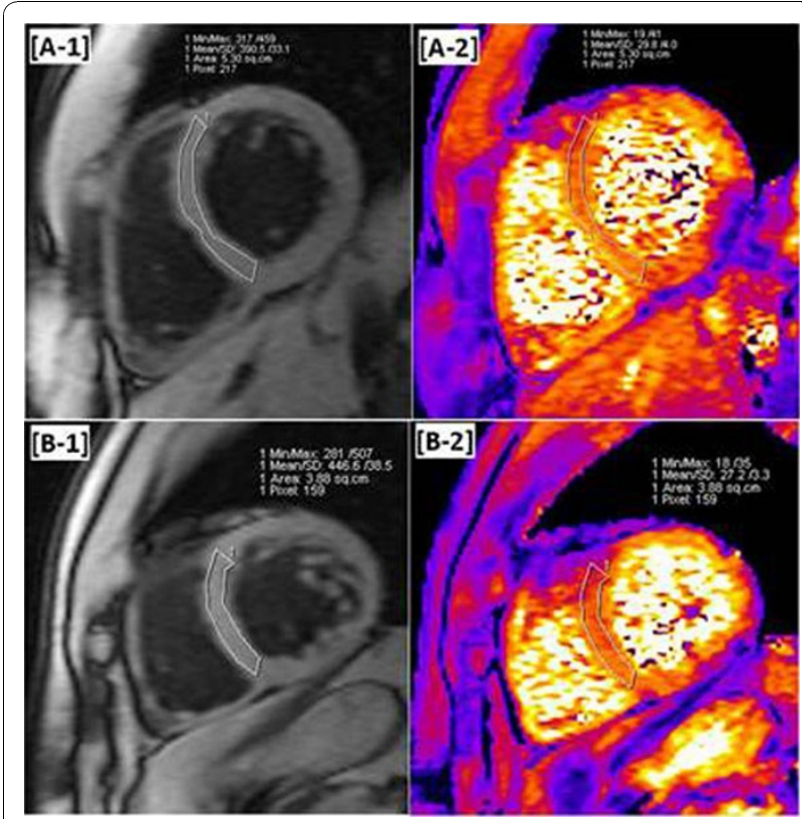

Figure 1 DB-prep GRE images [A-1, B-1] and corresponding T2*-map $[A-2, B-2]$ produced using inline analysis in two healthy volunteers. The contours of these images mark septal regions from which the average $T 2^{*}$ value was estimated. The average $T 2^{*}$ value within septal regions were $29.8 \pm 4.0 \mathrm{~ms}$ and $27.2 \pm 3.3 \mathrm{~ms}$ for these two subjects, which are significantly above $T 2^{*}<20 \mathrm{~ms}$ range indicating cardiac iron overload. 


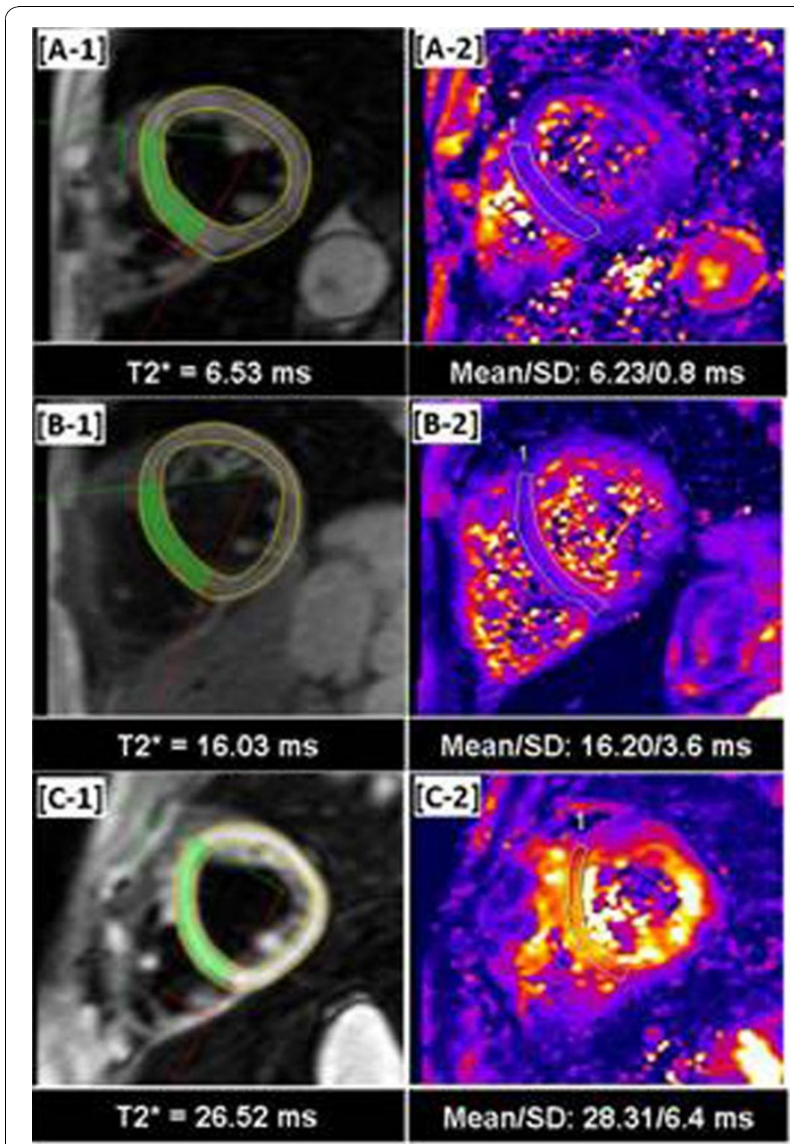

Figure 2 T2* estimates in 3 patients with suspected iron overload. $[A-1, B-1, C-1]$ A DB-prep GRE image showing the region used to T2* estimate within CMRTools. The estimated T2* is listed directly below each image. [A-2, B-2, C-2] Corresponding T2* maps obtained with inline analysis. Average of pixel-wise $\mathrm{T} 2^{*}$ estimate was obtained from indicated septal region. In all 3 cases, the average value obtained from $T 2^{*}$-map closely matches the one calculated using CMRTools. match closely with the values obtained using CMRTools. Fig. 3 illustrates statistical comparison of T2* estimates using CMRTools and inline analysis in all 32 patients.

\section{Conclusions}

The proposed technique computes pixel-wise T2* estimate which differs from region-based $\mathrm{T} 2^{*}$ assessment within CMRTools; however, the average T2* values within septum are highly correlated $(\mathrm{R} 2=0.996)$ with the region-based estimates obtained using CMRTools. This is an encouraging result given that $\mathrm{T} 2 \%$-map is generated on the scanner without any need for user intervention to eliminate outliers, and that assessment of myocardial $\mathrm{T} 2^{*}$ is possible immediately following data acquisition. Prospective clinical studies are warranted to thoroughly validate this proposed method.

\section{Author details \\ ${ }^{1}$ Siemens Healthcare, Chicago, IL, USA. ${ }^{2}$ Siemens Corporate Research, Princeton, NJ, USA. ${ }^{3}$ Siemens AG, Erlangen, Germany. ${ }^{4}$ Royal Brompton Hospital, London, UK.}

Published: 2 February 2011

\section{References}

1. Pennell DJ: Ann. N.Y. Acad. Sci. 2005, 1054:373-378.

2. He, et al: MRM 2008, 60:1082-1089.

doi:10.1186/1532-429X-13-S1-P308

Cite this article as: Shah et al.: Inline myocardial t2* mapping with iterative robust fitting. Journal of Cardiovascular Magnetic Resonance 2011 13(Suppl 1):P308.
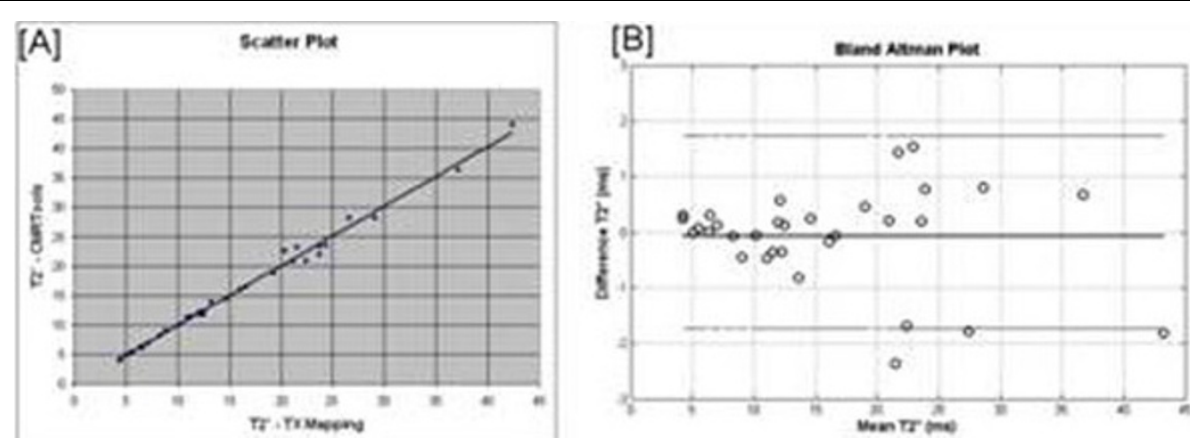

Figure 3 Statistical comparison between CMRTools and inline T2*-maps. 32 patients with suspected iron overload were retrospectively analyzed using both methods. The classification of each patient with severe $\left(\mathrm{T} 2^{*}<10 \mathrm{~ms} ; 10\right.$ patients), mild or moderate $\left(10 \mathrm{~ms}<\mathrm{T}^{*}<20 \mathrm{~ms}\right.$; 11 patients) or no iron overload $\left(\mathrm{T}^{*}>20 \mathrm{~ms} ; 11\right.$ patients) group was identical for both methods. [A] shows scatter plot compating T2* values estimated using CMRTools to those obtained using inline T2*-maps. Linear Regression: slope $=1.01$, intercept $=-0.12$ and $R^{2}-0.996$. [B] is a Bland-Altman plot compating T2* estimates using two methods within these patients. Dotted lines indicate $95 \%$ confidence intervals. 